\title{
A stepwise model for delivering medical humanitarian aid requiring complex interventions
}

\author{
J. Nilas Young, MD, ${ }^{\mathrm{a}}$ Josie Everett, BA, ${ }^{\mathrm{b}}$ Janet M. Simsic, MD, ${ }^{\mathrm{c}}$ Nathaniel W. Taggart, MD, ${ }^{\mathrm{d}}$ \\ Sonny Bert Litwin, MD, ${ }^{\mathrm{e}}$ Natalia Lusin, $\mathrm{PhD},{ }^{\mathrm{b}}$ Lizbeth Hasse, Esq, JD, ${ }^{\mathrm{f}}$ Evgeny V. Krivoshchekov, MD, ${ }^{\mathrm{g}}$ \\ James P. Marcin, MD, MPH, ${ }^{\mathrm{h}}$ Gary W. Raff, MD, ${ }^{\mathrm{i}}$ and Frank Cetta, $\mathrm{MD}^{\mathrm{d}}$
}

Objectives: Cardiothoracic surgeons and practitioners of cardiovascular medicine have a long history of humanitarian aid. Although this is worthwhile at multiple levels and occasionally described in some detail, few efforts have a proven algorithm with demonstrable outcomes that suggest effective educational methodology or clinical results approaching accepted standards in developed countries.

\begin{abstract}
Methods: Our report provides a stepwise approach to developing highly successful self-sustainable, replicable, and scalable humanitarian congenital cardiac surgical programs, and provides data to allow insight into the efficacy of our model.
\end{abstract}

Results: This program model has evolved over 25 years, during which it has been replicated several times and scaled throughout a vast and populous country. Since 1989, Russia has undergone considerable social, political, and economic changes. Our program model proved successful throughout this time despite dynamic social, political, and medical landscapes.

Conclusions: The positive results of our program model indicate that these methodologies may be helpful to others attempting to address the worldwide shortage of cardiovascular care and particularly the complex interventions required in the management of congenital cardiovascular disease. (J Thorac Cardiovasc Surg 2014;148:2480-9)

See related commentary on pages $2489-90$.

Supplemental material is available online.

Cardiovascular disease is the leading cause of mortality worldwide, and less than $15 \%$ of children with congenital heart disease (CHD) have access to medical care. Many substantive humanitarian efforts have been made to improve

\footnotetext{
From the Division of Cardiothoracic Surgery, ${ }^{a}$ University of California, Davis Medical Center, Sacramento, Calif; Medical Advisory Council, ${ }^{\mathrm{b}}$ Heart to Heart International Children's Medical Alliance, Oakland, Calif; Nationwide Children's Hospital, ${ }^{\mathrm{c}}$ Columbus, Ohio; Divisions of Pediatric Cardiology and Cardiovascular Diseases, ${ }^{\mathrm{d}}$ Mayo Clinic, Rochester, Minn; Herma Heart Center and Division of Cardiothoracic Surgery, ${ }^{\mathrm{e}}$ Children's Hospital of Wisconsin, Milwaukee, Wis; Creative Industry Law, LLP, ${ }^{\mathrm{f}}$ San Francisco, Calif; Department of Cardiovascular Surgery, ${ }^{\mathrm{g}}$ Research Institute of Cardiology, Tomsk Scientific Centre, Siberian Branch of the Russian Academy of Medical Sciences, Tomsk, Russia; Pediatrics Division, Critical Care, ${ }^{\mathrm{h}}$ University of California, Davis Medical Center, Sacramento, Calif; and University of California, Davis Medical Center, ${ }^{\mathrm{i}}$ Sacramento, Calif.

Heart to Heart International Children's Medical Alliance is a 501(c)(3) nonprofit humanitarian organization that has received contributions from a variety of donors.

Disclosures: Authors have nothing to disclose with regard to commercial support.

Received for publication May 8, 2014; accepted for publication July 27, 2014; available ahead of print Sept 26, 2014.

Address for reprints: J. Nilas Young, MD, Cypress Building, UC Davis Medical Center, 2221 Stockton Blvd, 2nd Floor, Room 2112, Sacramento, CA 95817 (E-mail: nilas.young@ucdmc.ucdavis.edu).

$0022-5223 / \$ 36.00$

Copyright (c) 2014 by The American Association for Thoracic Surgery

http://dx.doi.org/10.1016/j.jtcvs.2014.07.067
}

global cardiovascular care, and many more are needed. ${ }^{1-21}$ By drawing on a 25-year experience developing congenital cardiac surgery programs with an emphasis on newborns and infants-a highly complex management group-we have developed a successful program model for achieving high-quality outcomes, sustainability, scalability, and efficacy applied to a large geographic region with a large population base (Russian Federation). Although we have focused on a specific class of disease, our model may be applicable for other medical humanitarian projects, particularly those concerned with complex surgical interventions.

\section{HISTORY}

Beginning in 1988, our project was initiated and nurtured by that ever-common element in medical humanitarian enterprises - the quest by a parent for medical care for her invalid child - which resulted in contact with receptive lay and medical persons sympathetic to the patient's plight. The interactions resulted in transport to the United States and successful therapy for the patient. In our case, a 7-year-old girl had tetralogy of Fallot with end-stage physiology at a time in the Soviet Union when surgical therapy for her pathology was believed to be associated with excessive risk. A native of then Leningrad (now St Petersburg) Russia, that first patient is now an asymptomatic 32-year-old mother of a healthy 4-year-old boy.

That early successful outcome received widespread media coverage that ultimately resulted in her US physicians being invited to begin a congenital cardiac surgery program 


\section{Abbreviation and Acronym}

$\mathrm{CHD}=$ congenital heart disease

in Leningrad, Russia in 1989-at that time the second largest city in the Soviet Union. ${ }^{22,23}$ Subsequent to the development of a highly respected and well-recognized congenital cardiac program at Children's Hospital \#1 in Leningrad, our volunteer-driven organization (Heart to Heart International Children's Medical Alliance) has established 3 additional highly active congenital cardiac programs in 3 other major geopolitical regions of Russia. In addition, 2 other early-stage collaborations are currently under way (Figure 1).

Initiated at the end of the Cold War, and well received by our Russian colleagues, our programs have had major impacts on congenital cardiac care for the regions involved and the country as a whole. More than 16,000 children born with CHD in Russia have had congenital cardiac surgery or catheter-based interventions by cardiac specialists trained by Heart to Heart. Currently, each year approximately 1500 children receive surgical cardiac interventions at our established programs (Figure 2).

\section{HEART TO HEART PROGRAM MODEL}

Heart to Heart's strategic objective is to assist the Russian medical community in providing access to lifesaving heart care for all children in Russia by developing a network of self-sustaining cardiac centers of excellence in strategically located metropolitan areas. Heart to Heart continues to refine its program model as its medical volunteers continue to implement it throughout the Russian Federation.

\section{Step 1: Site Selection: Choosing a Site and Collaborative Partners}

Performing due diligence to select a site for development is a critically important first step. Heart to Heart makes a considerable investigative and financial investment in the process of choosing a new Russian team to train. Typically, the process is initiated by a Russian physician(s) or institution formally communicating to Heart to Heart an interest in obtaining assistance to develop a pediatric cardiac program.

\section{Step 2: Demographic Research}

Heart to Heart conducts initial research to assess the size of the local/regional population under consideration, air and ground transportation, and political stability. Site demographics characterized by insufficient patient referral bases, overly cumbersome transportation routes (to/from the United States), inadequate financial or physical infrastructure, or military conflict or potential major environmental hazards are excluded. Proximity to an established pediatric cardiac center also would be considered contrary to our program strategy.

\section{Step 3: Initial Site Assessment}

A small team (pediatric cardiologist or pediatric cardiac surgeon with international humanitarian aid experience and Heart to Heart's executive director) spends 2 to 3 days in the potential site city visiting the host medical institution and other medical institutions with collaborative potential (eg, children's hospitals, neonatal/maternity centers, adult cardiac centers). Meetings are held with key physicians, department chiefs, hospital administrators, and representatives of the regional ministry of public health and regional governor's office. The primary purpose of the first site

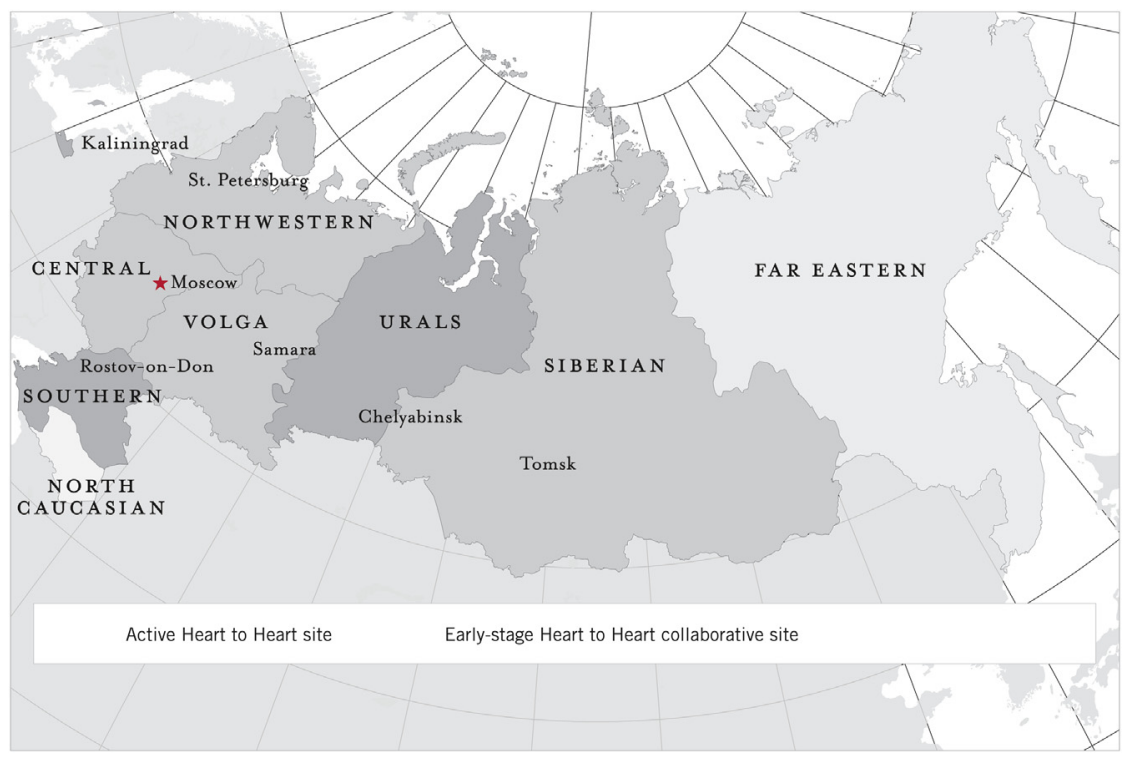

FIGURE 1. Heart to Heart self-sustaining and developing sites. The Russian Federation is made up of 8 geopolitical districts. Note: Heart to Heart's work does not extend to the Central District (home to Moscow and advanced cardiac programs) or the North Caucasian District (politically unstable). 


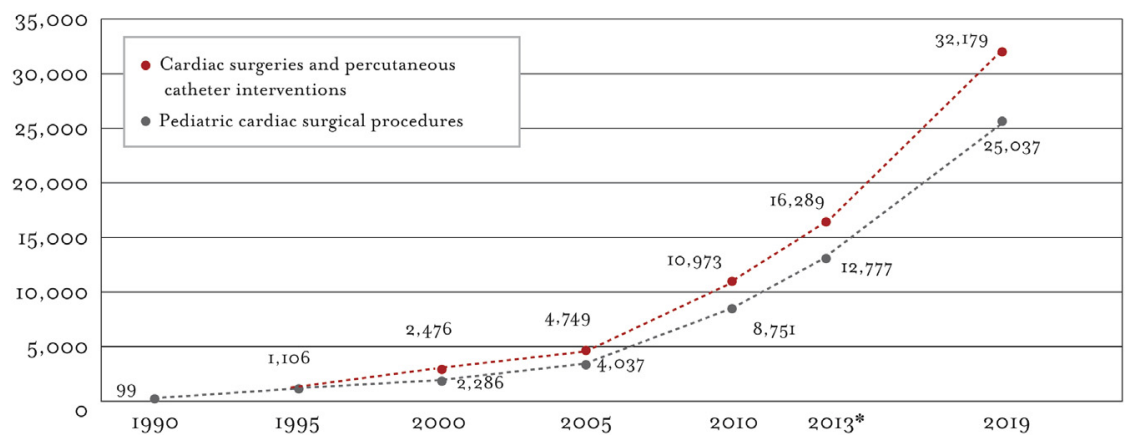

FIGURE 2. Pediatric cardiac case volumes at all of Heart to Heart's collaborative sites in Russia. Figure indicates projected volumes to 2019.

assessment visit is to determine whether adequate hospital equipment and personnel exist, and to evaluate whether there is a potential for program self-sustainability. The secondary purpose of the site assessment visit is to explain Heart to Heart's program model and to begin a discussion about the respective roles and responsibilities of key stakeholders. Heart to Heart's assessment team observes the local team performing cardiac surgery. The host hospital's cardiac surgical outcomes data are reviewed by the Heart to Heart team to help assess the host site's contemporary status and potential for complex congenital cardiac surgery.

Bringing complex medical care to an institution that has limited or minimal experience with similar disease processes is extremely challenging and requires a broad and thoughtful educational effort. Team building is critical from the outset, and the value of the many varied participants must be emphasized. Many institutional programs in foreign lands are extremely hierarchal, and many (possibly all) medical participants may be very poorly salaried. Heart to Heart carefully assesses the level of capability of the host institution, which typically requires at least 2 site visits. Hospital and medical administration, physicians, and in particular the head of the host team must strongly support the program or the effort is doomed to failure. We have noted that some physicians have dual roles (eg, anesthesiology and perfusion), which can complicate team building.

\section{Step 4: Secondary Site Assessment}

If the first site assessment team identifies potential for successful program development and our Board of Directors concurs, then a second small team (of similar composition) travels for a several day site assessment to the host medical institution and continues dialogue with local medical leaders and key stakeholders. We hold meetings to ascertain the commitment of local stakeholders, including hospital administrators and aspiring pediatric cardiac team members, and separately with regional health officials with the goal of determining whether sufficient political will and economic resources exist to achieve selfsustainability. If it appears that both Heart to Heart and the Russian medical institution are enthusiastic about a joint effort, then a formal protocol with bilateral commitments is signed by medical, administrative, and governmental parties during or after the second visit but before any further visits are made.

Choices for site assessment continue to evolve. Fortunately, the Russian government has recently provided funding for the establishment of designated federal cardiac centers throughout the country that will eventually include the full spectrum of cardiac surgical services, including congenital cardiac surgery. Heart to Heart is currently collaborating with several of these centers and assessing their needs and potential for development as advanced congenital cardiac care programs.

\section{Step 5: Constructing Surgical-Educational Teams}

Heart to Heart recruits select teams of pediatric cardiac specialists to travel to Russia to conduct surgicaleducational missions. Generally speaking, Heart to Heart teams consist of 12 to 15 persons. The guest/US surgeon is encouraged to choose and invite operating room team members (pediatric cardiac anesthesiologist, perfusionist, and operating room technician) and several cardiologists. Remaining team members include a pediatric intensive care physician and 2 to 3 nurses, and 1 to 3 administrative support staff and interpreters. For subsequent medical missions, other specialists (eg, neonatologists) are recruited depending on the educational objectives for any given program year. Ideally, and on occasion, the entire team would come from a single institution, thereby providing optimum continuity. The logistics for accomplishing these team groupings and transportation for all specialists from multiple institutions, including visa procurement and team safety assurances, can be involved (Figure 3).

Heart to Heart's medical volunteers. Several criteria are used to select medical volunteers to implement our program model. Medical volunteers should (1) be employed as a pediatric cardiac specialist at an established medium- or highvolume center (nearly all Heart to Heart medical volunteers are US based); (2) be interested in and able to teach specific skills or lecture on specific topics; (3) possess a clear understanding that Heart to Heart's objective is for the local team 


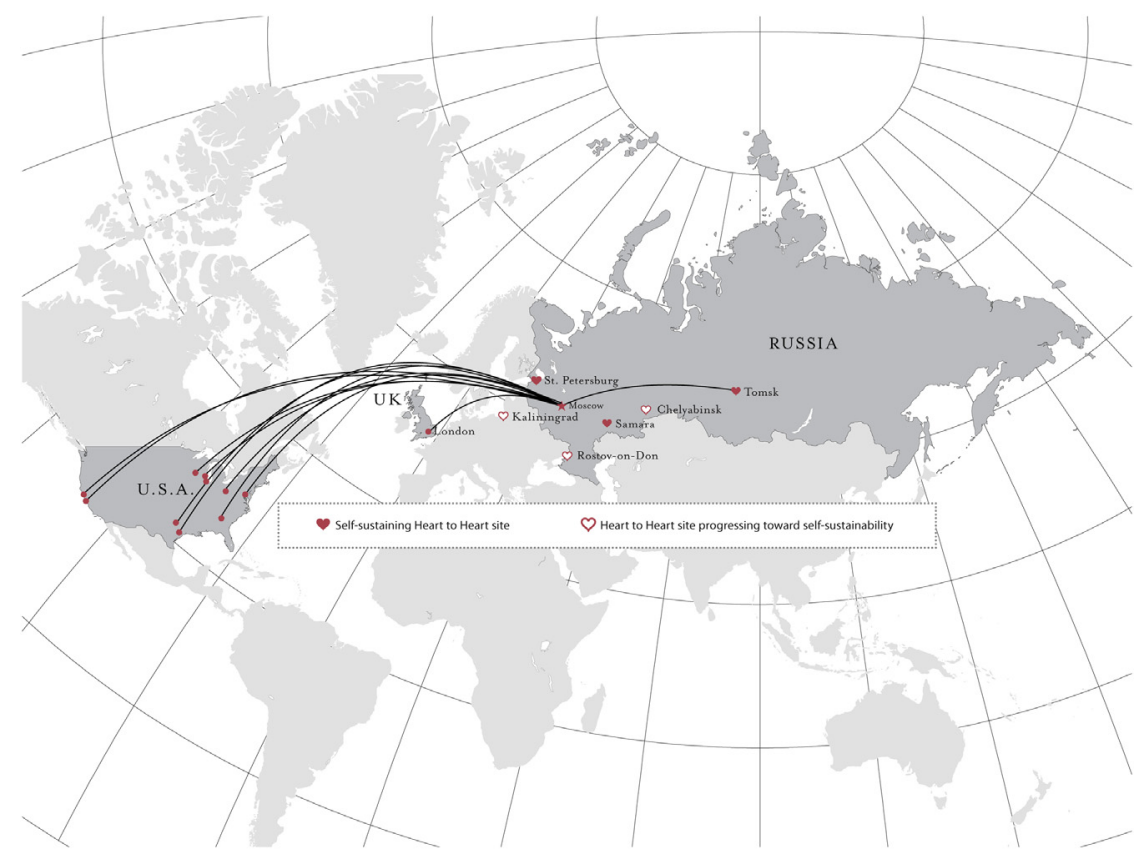

FIGURE 3. Coordinated travel routes of Heart to Heart specialists for missions to Tomsk, Siberia, 2006-2012. Medical volunteers represent the following institutions: Berlin Heart Inc, Spring Tex; Children's Healthcare of Atlanta/Emory University Hospital, Atlanta, Ga; Children's Hospital of Philadelphia, Philadelphia, Pa; Children's Hospital of Wisconsin, Milwaukee, Wis; Children's Medical Center of Dallas, Dallas, Tex; The Hospital for Sick Children, London, UK; Loyola University Medical Center, Maywood, Ill; Mayo Clinic, Rochester, Minn; Nationwide Children's Hospital, Columbus, Ohio; UC Davis Medical Center, Sacramento, Calif; UC San Francisco Children's Hospital, San Francisco, Calif.

to achieve self-sustainability within 5 to 7 years; and (4) be committed to developing a climate of teamwork and adherence to our program model, which may differ from that of their own institutional paradigms.

A major role of the Heart to Heart team is to demonstrate and perpetuate a climate of teamwork. Foreign medical systems are sometimes fragmented because of the diversity of training backgrounds. It is essential to demonstrate how practitioners from different subspecialties and disciplines can work through problems together. The goal of our teaching is that the Russians continue to work cohesively as a team for the good of the patients after we leave.

\section{Step 6: Annual Surgical-Educational Missions: Essentials of Information Transfer}

Our typical surgical-educational mission sends a small advance team of 2 cardiologists and 1 to 2 support staff who arrive at the partner site for 3 to 4 days before the full team unites on the ground. The primary task of the advance team is to examine patients-while teaching diagnostic methodology — and begin selecting surgical candidates for the joint Heart to Heart-Russian team to treat. To implement Heart to Heart's program model, the advance team must clearly understand their role in relation to the educational objectives for the current program year and the particular surgical-educational mission.
The clinical agenda for each medical mission is determined by joint agreement between the Russian and American team leaders, and is geared toward a long-range view of managing progressively complex pathologic entities. Each mission focuses on a certain subset of congenital cardiac pathologies, determined by pre-trip evaluation of the host center's experience and selected to provide teaching opportunities that will promote an appropriate positive growth experience for the host program, for example, tetralogy of Fallot.

One of the more difficult aspects of implementing our program model is dealing with the reality that we cannot save every child we examine. Not all volunteers are suited for this psychologically challenging and recurring aspect of on-site involvement, but it is critically important that the majority of patient cases chosen for surgery provide the opportunity for Heart to Heart to effectively teach whatever skills and knowledge have been agreed on to be the current year's educational objectives. If the latter is not accomplished, the local team's learning stagnates and program progress is limited.

Effective transfer of information from teacher to learner depends on a number of factors: recognition of knowledge deficits of the learner, expertise of the teacher, and a teaching style that are appropriate for both the topic of discussion and the learner's level of understanding and the opportunity for clarification, evaluation, and correction. The mentor 
often finds himself/herself teaching in several distinct clinical scenarios: bedside patient evaluation, echocardiography, cardiac catheterization, the intensive care unit, and the operating room. In this context, 2 unique obstacles to clear and efficient communication are evident: (1) language barriers and (2) the unique subspecialty training and organizational structure of the Russian host team.

Language barriers extend beyond a basic need for translation from English to Russian and vice versa. Medical terminology is fraught with acronyms, abbreviations, colloquialisms, and nuance, each of which can quickly lead to misunderstanding and misinformation. Overcoming these language barriers requires interpreters who are facile in both English and Russian. Most native Russian interpreters, though, have a limited knowledge of medical terminology, let alone a conceptual understanding of congenital cardiology or cardiac surgery. Therefore, part of the responsibility for ensuring clear communication falls on the Englishspeaking teacher.

Our interpreters are provided with lectures and materials on basic cardiac anatomy and physiology. The interpreters often have nontechnical backgrounds, so visual aids used to describe anatomy and cardiac function are essential. The teachers must be able to explain medical concepts and terminology such that they can be understood by nonmedical interpreters. Professional oversight of interpreters is critical to effective communication.

A second obstacle to clear and efficient transfer of information particular to Heart to Heart surgical-educational missions arises from the unique specialty structure of the Russian congenital heart team. As an example, in the United States, physicians who perform echocardiography and cardiac catheterization are primarily trained as general congenital cardiologists. In Russia, because of abbreviated training for specialists, time is not allotted for general cardiology training before specialization. As a result, although Russian echocardiographers are highly skilled at the technical aspects of cardiac ultrasound, there tends to be a lack of practical understanding of cardiac hemodynamics, anatomy, and treatment options. Conversely, Russian general cardiologists often lack a functional understanding of echocardiography. This division of duties often can lead different specialists to function independently of each other, which in turn leads to poor or incomplete communication of patient information.

Transfer of knowledge and skills at the surgical level is particularly complex. No surgeon trainee is able to observe every operation he or she will be expected to perform. Demonstrating a complex surgical procedure is the ultimate surgical mentorship. Surgical trainees are allowed to see nuances of an operation that can also be applied to multiple other procedures. Building on this experience to develop confidence is incomparable in the development of a surgeon's success. The role and responsibilities of the lead surgeon for advancing the entire operative team's capabilities and performance are emphasized. In addition to intraoperative and postoperative care techniques, our team demonstrates methods of patient selection, that is, choosing the right operation for the right patient at the right time. This is a most important aspect of congenital heart surgery and the major determinant of success versus failure. Therefore, the types of cases selected are far more important than the number of cases performed during a visit. Typically, 1 case per day will be performed over a 1- to 2-week educational mission. What is important is not how many cases the joint Russian-Heart to Heart team performs, but rather the number and outcomes of the cases performed by the Russian team after Heart to Heart visits.

Our surgical-educational missions are also opportunities for further program evaluation. We emphasize a "communication feedback loop." During these missions, we emphasize daily joint case conferences. On the basis of data review and empirical observations on the ground, Heart to Heart team members are asked to lead a "wrap up" meeting at the end of each mission, during which members of the medical teams are asked to comment on the local team's current knowledge base, skill set, team work, systems, and equipment and supplies, and to provide "next steps" for the local team to implement over the next 12 months.

After the visiting US team returns home, the Heart to Heart medical team leadership and staff generate a written report of these recommendations. The report is submitted to the chief of the Russian program and their department heads in English and Russian. The report is broken down to provide specific recommendations to all specialists on the local team by subspecialty: pediatric cardiac cardiology, surgery, anesthesia, nursing, and intensive care.

\section{Step 7: Program Evolution and Maturation}

Development of a complex humanitarian aid program requires an initial conservative approach to care or procedures. This approach results in a higher level of success not only in patient outcomes but also in the absolute transfer of medical knowledge, which is what will ultimately enable the host institution's medical practitioners to replicate the care patterns and outcomes of the visiting humanitarian aid team. In the case of pediatric cardiac surgery, ensuring the presence of solid basic diagnostic procedures, for example, echocardiography and clear treatment plans for straightforward operations (eg, surgical repair of patent ductus arteriosus, coarctation, atrial septal defect, and ventricular septal defect), is critical to further the host team's progress. In reality, these procedures can palliate and cure a large percentage of patients with congenital heart defects and do not place severe burdens on limited resources. Once successful outcomes start to occur, more complex lesions can be addressed, assuming the financial and infrastructure support required for their successful management can keep pace. 


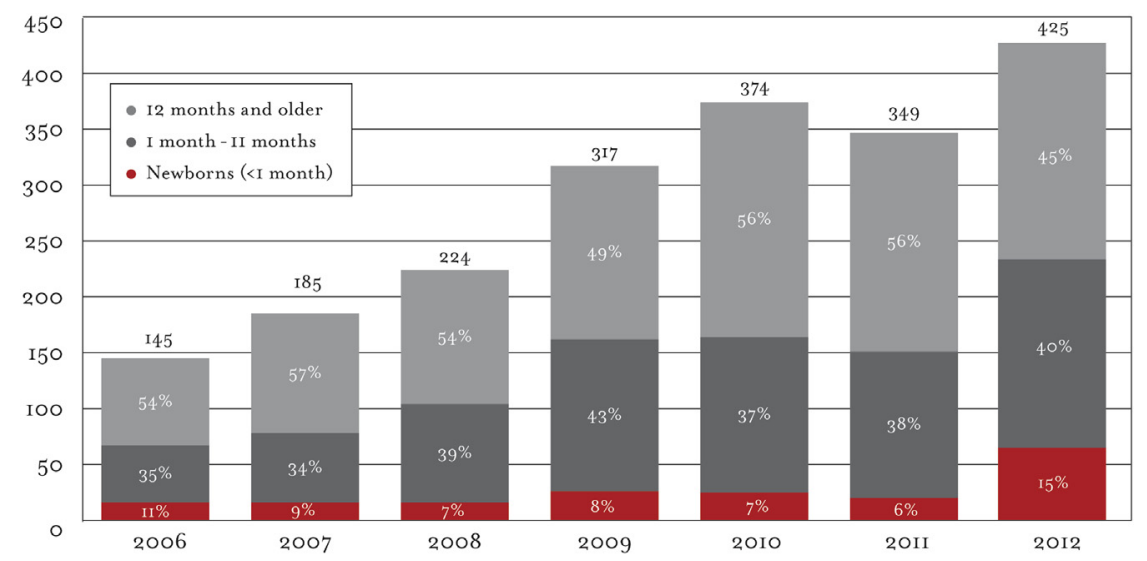

FIGURE 4. Pediatric cardiac surgical case volumes by year and age group at Heart to Heart's Tomsk site 2006-2012. Note progressive increase in younger patients, including newborns.

In our experience, a 5- to 7-year process is typically involved to allow for high-quality self-sustaining programs to be developed. With the advent of the internet over the past 25 years, we have been able to establish good datasharing mechanisms with our respective host institutions that include preoperative and postoperative data, as well as outcomes results analysis. The availability of internet technology also allows the Heart to Heart and Russian teams to share diagnostic studies, including complex imaging (eg, echocardiograms), and makes preoperative planning and postoperative consultation facile for host and visiting team members.

An example of our anticipated trajectory for program maturation can be appreciated by reviewing the progress over time of our recent educational collaboration in Tomsk, Siberia, our third pediatric site in Russia (Figures 4-6). During Heart to Heart's 7-year involvement in Tomsk, there was a dramatic increase in case volumes simultaneous to a progressively higher level of case complexity, and the outcomes have been superb.

\section{Step 8: Educational Outreach and Interactive Support Between Developed and Developing Programs}

We have respectfully promoted and have been pleased with the cross-fertilization of our programs. For example, surgeons from our established programs have participated in our team trips to developing sites. Established and developing programs have always welcomed, and we have encouraged, visiting medical personnel from any institution interested in observing our team(s) in action. Frequently, 20 to 30 visiting medical personnel from other Russian programs have attended our educational team trips (Figure 7).

\section{Role of Data Collection and Analysis}

It is critically important to have the host institution invested in high-quality outcomes that can be readily measured and case analyses that can be quickly shared. This requires implementation of database tools that are frequently absent in developing medical programs. The metrics used by Heart to Heart to evaluate programmatic

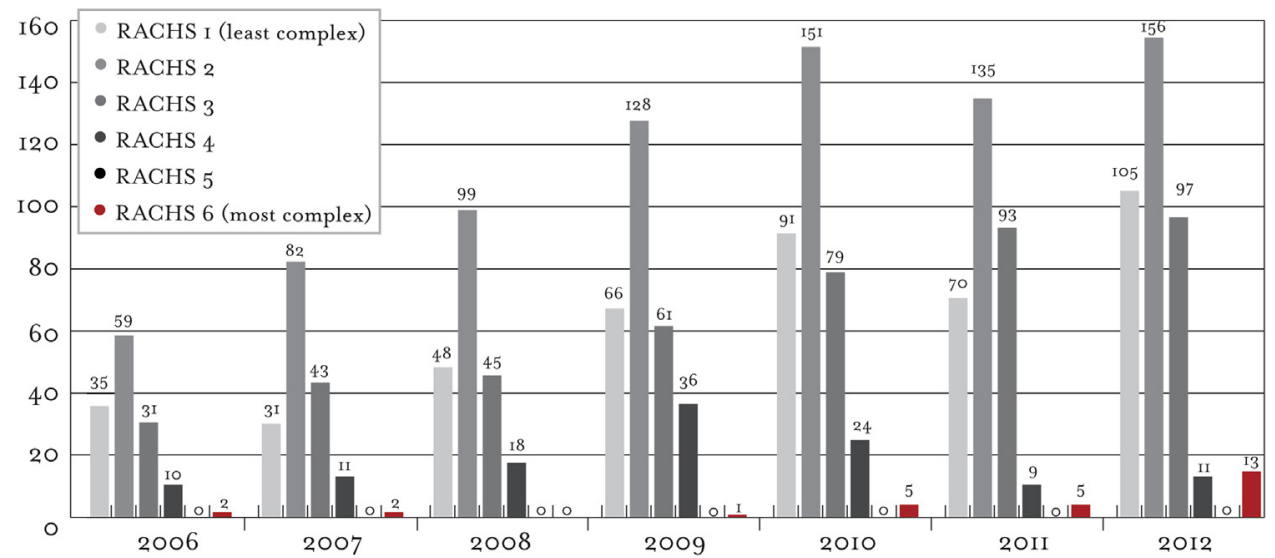

FIGURE 5. Pediatric cardiac cases (classifiable by Risk Adjustment for Congenital Heart Surgery 1 scores) by year and procedure complexity at Heart to Heart's Tomsk site 2006-2012. Note volume increase in procedures performed at every level of complexity. RACHS, Risk Adjustment for Congenital Heart Surgery. 


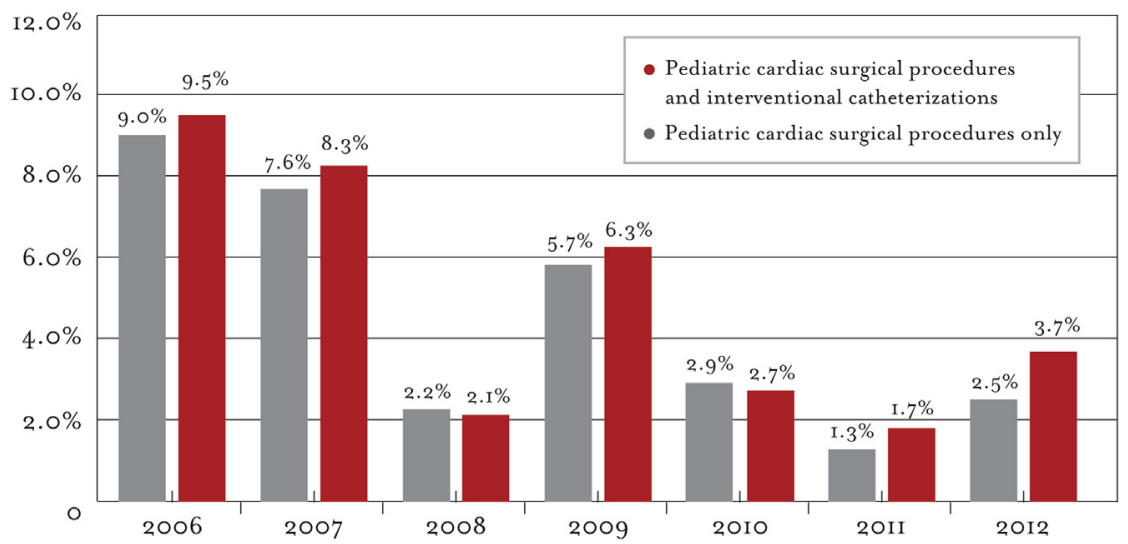

FIGURE 6. Mortality for pediatric cardiac surgical procedures: Tomsk, 2006-2012. Note marked decrease in operative mortality over time in conjunction with a progressively higher level of case complexity (Figure 5).

progress and achievement of self-sustainability are currently surgically based, but catheter-based interventions are also rigorously documented and include patients who have had surgery, interventional catheterizations, diagnostic catheterizations, echocardiograms, and outpatient examinations in the time intervals being analyzed. Data are provided by the Russian cardiac center and include all of their cases from newborn to 17-year-old patients.

By using our ongoing communications feedback loop, Risk Adjustment for Congenital Heart Surgery 1 methodology as a guide and a metric, and CardioAccess (CardioAccess Inc, Fort Lauderdale, Fla) and Microsoft Excel (Microsoft Corp, Redmond, Wash) to track surgical outcomes, we critically review outcomes in detail with our Russian teams, assess results on the basis of contemporary benchmarks, and relate these to our programs' situational circumstances. ${ }^{24-28}$

\section{Role of Strategic Guidance and Leadership Development}

As with all surgical and medical training programs, a major goal is to enable the host teams to manage cases of graded complexity. Heart to Heart's Medical Advisory Council, administrative staff, and medical volunteers work together to review surgical outcomes data, and by collaborating with our Russian colleagues, set and monitor annual educational objectives for each team we are training. As with all medical organizations, personnel issues and vacancies arise with some frequency at the host institutions. Good leadership at all levels of a developing program is

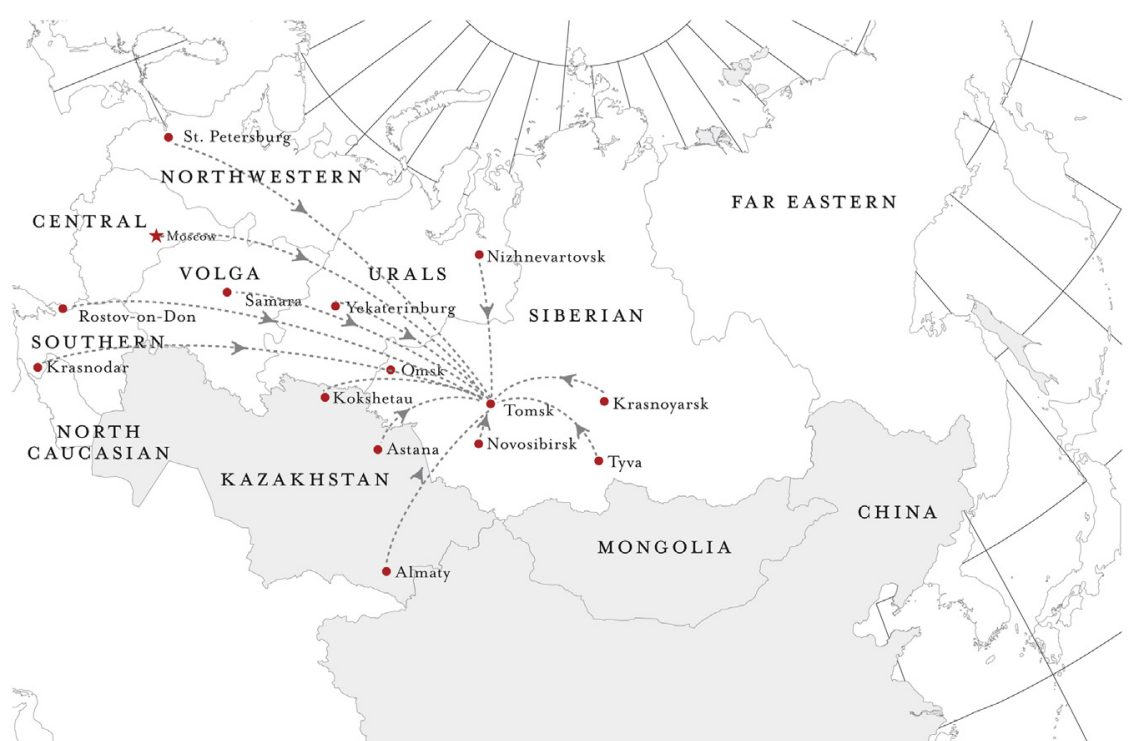

FIGURE 7. Russian medical colleagues from various cities observed Heart to Heart's surgical-educational mission in Tomsk, Siberia, 2011. Arrows depict travel routes from cardiac programs throughout Russia sending medical personnel. In this instance, a total of 35 specialists traveled to Tomsk, Siberia, to observe our educational collaboration. 
extremely important, and when deficiencies occur, for whatever reason, Heart to Heart takes an active consultative role to correct significant gaps in leadership, personnel, or team performance.

\section{Role of Scholar Exchange and Continuing Education}

Since establishing Heart to Heart, we have provided financial support for the host Russian institution surgeons, cardiologists, intensivists, and anesthesiologists to visit centers of excellence in the United States. On occasion, we have invited hospital administrators. This is an extremely valuable experience and one that allows the host team members to "put everything together" regarding Heart to Heart teams' rationale and conduct. Ideally, these hosted trips extend over several weeks, and the visiting medical personnel are sent to 1 and preferably 2 centers of excellence. A funded "traveling scholars" fellowship has been created by our organization to ensure continuity of this important educational function. Heart to Heart's medical volunteers and staff actively promote quality research projects collaboratively with our Russian colleagues, including abstract and manuscript production for international meetings. We promote educational conferences locally and on a regional/national level.

\section{Role of Equipment and Supplies}

Early in our involvement, we were required to transport with our traveling team much of the surgical instrumentation and medical devices (ventilators, monitors, echocardiography, and perfusion apparatuses) needed to safely accomplish surgical and interventional procedures. This requirement has decreased considerably and is currently negligible. We do believe it is best to perform cases using the host institution's equipment and supplies, so that the host team can continue to perform such cases after we leave. This also makes obvious any advantages and limitations of the host institution's equipment to all involved.

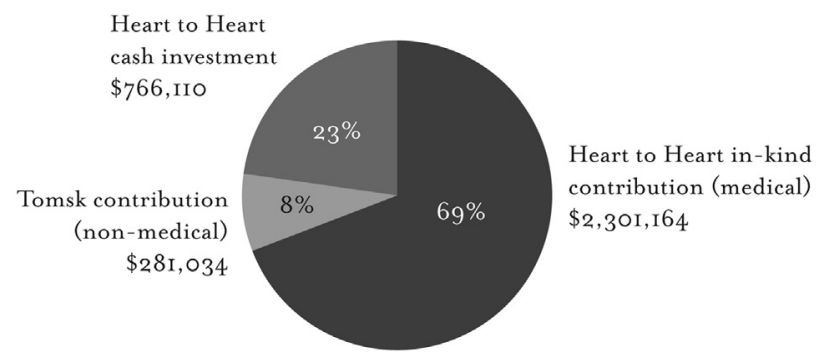

FIGURE 8. Heart to Heart's program value. The chart shows the actual total expenses needed to implement Heart to Heart's program model in Tomsk, Siberia, 2006-2012. Total program value is the sum of nonmedical in-kind donations, in-kind medical services (pro bono work of medical volunteers), and actual expenses (travel, lodging, visas, and amortized Heart to Heart staff time and services) in US dollars.

\section{Organizational Structure of a Medical Humanitarian Entity: Ensuring Financial Solvency and Legal Security at Home and Abroad}

In addition to emphasizing excellent outcomes for our clinical programs, we also emphasize that the financial leverage provided by our humanitarian aid program is substantial, at a ratio of approximately $\$ 3$ of additional program value generated for every $\$ 1$ of expense (Figure 8).

In the case of Heart to Heart, organizational financial sustainability is dependent on considerable volunteerism. All of our medical volunteers, board of directors, medical advisory board, chief statistician, and many important nonmedical personnel are volunteers. Without the work of these nonpaid participants, the organization could not carry out its mission. Heart to Heart has benefitted tremendously from the consistent team work of a tightly knit, hands-on board of directors who take a long view, including the continuous active leadership of a founding medical director. In addition, the organization is dependent on highly skilled management provided by a seasoned nonprofit executive director and a professionally trained small staff.

The organization, a 501(c)(3) charitable nonprofit corporation with federal tax-exempt status, acts with transparency and relative simplicity so that all stakeholders (both foreign and US based) can readily understand and trust its operations and messaging. To operate in an environment that presents unknowns and often lacks predictability, Heart to Heart's structure must be at once durable and flexible. The key to success for international nonprofit projects at all stages of evolution is in ensuring that the public can readily ascertain the effectiveness of the organization in carrying out and actually achieving its stated mission (Appendix E1).

\section{Limitations}

There are numerous limitations in attempting to evaluate the impact and efficacy of humanitarian programs, including those of Heart to Heart's:

1. Data were submitted to Heart to Heart by the various Russian cardiac centers, introducing possible bias.

2. Early in the program, data collection was problematic, but with improved internet access has become more reliable.

3. Mortality was calculated as deaths per procedure, not deaths per patient or hospitalization, which resulted in slightly lower mortality percentages.

4. With the changing Russian landscape, our programs have emphasized comprehensive congenital disease management with a focus on newborn pathologies. The burgeoning Russian Federal commitment to cardiac care for its populations will have major impacts on this effort. 


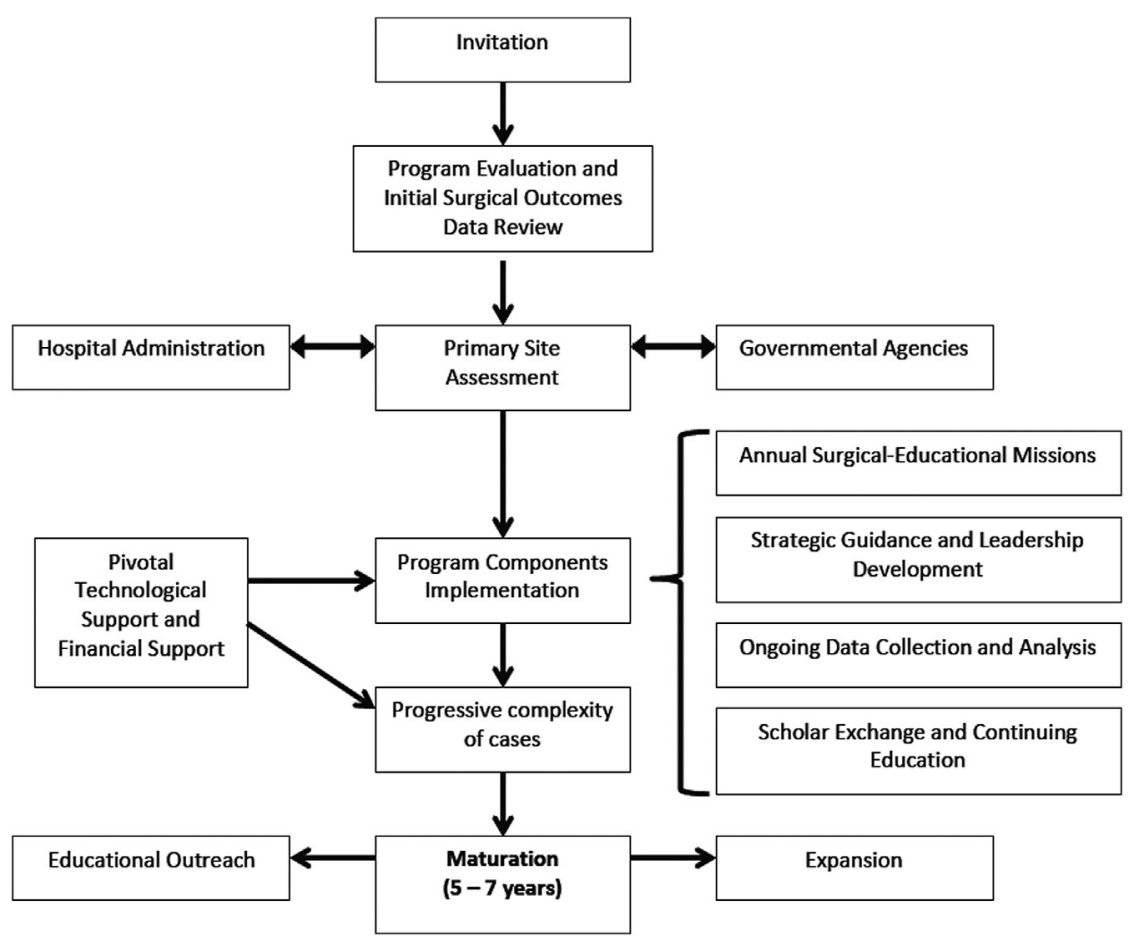

FIGURE 9. Heart to Heart site development algorithm.

5. The realities of our reliance on volunteer US medical personnel from different institutions impairs Heart to Heart's ability to deliver a $100 \%$ uniformly consistent teaching style and message. However, concerted efforts to provide as much consistency as possible has resulted in relatively quick advancement to self-sustainability for each of our cardiac programs over the last 25 years.

\section{CONCLUSIONS}

The worldwide need for and benefit of medical humanitarian aid for patients with CHD has become increasingly evident. ${ }^{29,30}$ Our successful program model has evolved over 25 years. We believe this model is an effective way of teaching how to save the lives of children born with heart disease in areas of need and has the potential for expansion to multiple geographic regions (Figure 9). We focus on teaching lifesaving surgical and medical technology to treat all children with congenital heart defects, from the most complex newborns to the most complex adolescents. As the adult congenital population grows in Russia, Heart to Heart has expanded its focus to include support of the St Petersburg adult CHD program. The program model that Heart to Heart uses reflects the evolution of contemporary congenital heart surgery. We emphasize the critical necessity of a coordinated team approach that engages the whole spectrum of medical, administrative, and civic partners needed to ensure high-quality outcomes, self-sustainability, scalability, and economic efficiency.

\section{References}

1. Yacoub MH. Establishing pediatric cardiovascular services in the developing world: a wake-up call. Circulation. 2007;116:1876-8.

2. World Health Organization. Global Status Report on Non-Communicable Diseases 2010. Available at: http://www.who.int/cardiovascular_diseases/ resources/atlas/en. Accessed September 19, 2014.

3. World Health Organization. Global Atlas on Cardiovascular Disease Prevention and Control. Available at: http://www.who.int/cardiovascular_diseases/ resources/atlas/en. Accessed September 19, 2014.

4. Larrazabal LA, Jenkins KJ, Gauvreau K, Vida VL, Benavidez OJ, Gaitán GA, et al. Improvement in congenital heart surgery in a developing country: the Guatemalan experience. Circulation. 2007;116:1882-7.

5. Jonas RA. Congenital heart surgery in developing countries. Semin Thorac Cardiovasc Surg Pediatr Card Surg Annu. 2008;3-6.

6. Giamberti A, Mele M, Di Terlizzi M, Abella R, Carminati M, Cirri S, et al. Association of Children with Heart Disease in the World: 10-year experience. Pediatr Cardiol. 2004;25:492-4.

7. Magee WP Jr. Evolution of a sustainable surgical delivery model. J Craniofac Surg. 2010;21:1321-6.

8. Macfarlane SB, Agabian N, Novotny TE, Rutherford GW, Stewart CC, Debas HT. Think globally, act locally, and collaborate internationally: global health sciences at the University of California, San Francisco. Acad Med. 2008;83:173-9.

9. Koplan JP, Baggett RL. The Emory Global Health Institute: developing partnerships to improve health through research, training, and service. Acad Med. 2008; 83:128-33.

10. Chapin E, Doocy S. International short-term medical service trips: guidelines from the literature and perspectives from the field. World Health Popul. 2010; $12: 43-53$.

11. Ho TC, Ouyang H, Lu Y, Young AH, Chintala K, Detrano RC. Postprocedural outcomes of rural children undergoing correction of congenital heart lesions in Yunnan Province, China. Pediatr Cardiol. 2011;32:811-4.

12. Hughes C, Zani S, O'Connell B, Daoud I. International surgery and the University of Connecticut experience: lessons from a short-term surgical mission. Conn Med. 2010;74:157-60.

13. Jeuland M, Whittington D. Cost-benefit comparisons of investments in improved water supply and cholera vaccination programs. Vaccine. 2009;27: 3109-20. 
14. Johnson NL, Livingston MJ, Novak TS. A cost analysis of training expenses versus the value of medical care provided during West Africa Training Cruise 2004: Senegal. Mil Med. 2006;171:1225-8.

15. Velebit V, Montessuit M, Bednarkiewicz M, Khatchatourian G, Mueller X, Neidhart P. The development of cardiac surgery in an emerging country: a completed project. Tex Heart Inst J. 2008;35:301-6.

16. Cox JL. Presidential address: changing boundaries. J Thorac Cardiovasc Surg. 2001;122:413-8.

17. Pezzella AT. Progress in international cardiac surgery: emerging strategies. Ann Thorac Surg. 2001:71:407-8.

18. Ghosh P. Setting up an open heart surgical program in a developing country. Asian Cardiovasc Thorac Ann. 2005;13:299-301.

19. Bernier PL, Otal N, Tchervenkov CI, Jacobs JP, Stellin G, Kurosawa H, et al. An invitation to the medical students of the world to join the global coalition to improve care for children and adults with congenital heart disease across the world. Mcgill J Med. 2008;11:185-90.

20. Novick WM, Stidham GL, Karl TR, Guillory KL, Ivanćan V, Malcić I, et al. Are we improving after 10 years of humanitarian paediatric cardiac assistance? Cardiol Young. 2005;15:379-84.

21. Neirotti R. Access to cardiac surgery in the developing world: social, political and economic considerations. Presented at the FAC-Federacion Argentina de Cardiologia 5th International Congress of Cardiology on the Internet 5th Virtual Congress of Cardiology-QVCC; August 8, 2007. Available at: http://www.fac. org.ar/qcvc/llave/c010i/neirottir.pdf. Accessed September 23, 2014.
22. Young JN, Hardy CE, Helton JG. Circumstance, petition, and involvement in pediatric cardiac care in St. Petersburg, Russia 1988-1992. California Pediatrician. Am Acad Pediatr, California District. Fall 1992;10-5

23. Young JN. Heart to Heart - 10 years of cardiac surgery in St. Petersburg, Russia STS News. 1999;4.

24. Jenkins KJ, Gauvreau K, Newburger JW, Spray TL, Moller JH, Iezzoni LI Consensus-based method for risk adjustment for surgery for congenital heart disease. J Thorac Cardiovasc Surg. 2002;123:110-8.

25. DeCamp M. Ethical review of global short-term medical volunteerism. HEC Forum. 2011;23:91-103.

26. Eberlin KR, Zaleski KL, Snyder HD, Hamdan US. Medical Missions for Children. Quality assurance guidelines for surgical outreach programs: a 20-year experience. Cleft Palate Craniofac J. 2008;45:246-55.

27. Dearani JA. Improving pediatric surgical care in developing countries: matching resources to need. Semin Thorac Cardiovasc Surg Pediatr Card Surg Ann. 2010 $13: 35-43$.

28. Jacobs JP, Jacobs ML, Austin EH 3rd, Mavroudis C, Pasquali SK, LacourGayet FG, et al. Quality measures for congenital and pediatric cardiac surgery. World J Pediatr Congenit Heart Surg. 2012;3:32-47.

29. Hoffman JIE. The global burden of congenital heart disease. Cardiovasc J Afr. 2013;24:141-5.

30. Neirotti R. Paediatric cardiac surgery in less privileged parts of the world. Car diol Young. 2004;14:341-6.

\title{
EDITORIAL COMMENTARY
}

\section{Humanitarian congenital heart surgery: Template for success}

\author{
Carl Lewis Backer, MD
}

See related article on pages $2480-9$.

In this issue of the Journal, Young and coauthors ${ }^{1}$ have summarized for us a 25-year project that has resulted in the establishment of six new congenital heart surgery programs in Russia. All these were sites that had active adult cardiac surgical programs but lacked congenital cardiac surgery. The article is truly a template for success for groups that would like to provide a similar service in other countries. In fact, this is the primary mission of the World Society for Pediatric and Congenital Heart Surgery. Its vision statement affirms, "Every child born anywhere in the world with a congenital heart defect should have access to appropriate medical and surgical care. The mission of the World

From Ann \& Robert H. Lurie Children's Hospital of Chicago, Chicago, Ill. Disclosures: Author has nothing to disclose with regard to commercial support. Received for publication Sept 8, 2014; accepted for publication Sept 10, 2014; available ahead of print Oct 5, 2014.

Address for reprints: Carl Lewis Backer, MD, Ann \& Robert H Lurie Children's Hospital of Chicago, 225 E Chicago Ave, mc 22, Chicago, IL 60611-2605 (E-mail: cbacker@luriechildrens.org).

J Thorac Cardiovasc Surg 2014;148:2489-90

$0022-5223 / \$ 36.00$

Copyright (c) 2014 by The American Association for Thoracic Surgery http://dx.doi.org/10.1016/j.jtcvs.2014.09.013
Society is to promote the highest quality comprehensive care to all patients with congenital heart disease, from the fetus to the adult regardless of the patient's economic means with an emphasis on excellence in teaching, research, and community service.",

The authors of this article and the members of the Heart to Heart International Children's Medical Alliance should be congratulated on moving toward this goal in the vast country of Russia. The numbers alone are a dramatic measure of their success. In 1990, they started with only 99 patients undergoing surgical or transcatheter interventions. This has grown to more than 16,000 cumulative procedures through 2013, with ever decreasing mortality! The article is filled with "pearls" that, when followed, result in selfsustaining centers that perpetuate a climate of team work and continue to perform cases successfully after the visits from the Heart to Heart Alliance end. Young and coauthors also provide us with a timeline for success, a 5- to 7-year period that, in many respects, is not unlike the 5 to 7 years that it takes to train a cardiac surgeon after medical school.

I would encourage anyone who wants to embark on such a humanitarian mission to read this article as a template for success. Young and coauthors provide eight separate steps that they believe will lead to success in attaining 


\section{APPENDIX E1. FINANCIAL SOLVENCY}

Providing financial solvency for a humanitarian organization may be the most difficult aspect of accomplishing a persevering organizational effort. A large variety of successes have been achieved via a spectrum of fund-raising mechanisms, but ultimately it is a professional exercise that requires dedicated and highly competent individuals.

\section{LEGAL CONSIDERATIONS: BASIC LEGAL NEEDS OF INTERNATIONAL MEDICAL PROJECT}

Currently, there are no established legal guidelines for structuring a sustainable cross-border surgical training and treatment program. Heart to Heart's legal underpinnings are grounded on the US federal 501(c)(3) organizational structure that has proven reliable for all involved: patients and their families, volunteer medical practitioners in the United States, professionals in the beneficiary medical institutions, other volunteers, institutional funders, individual donors, staff, officers and directors, and the public. This flexible nonreligious and apolitical structure, with its transparency in funding and accounting, has been crucial to maintaining the organization's activities through various stages of growth and well-being. The primary demands on the Heart to Heart organizational structure are that it provide the framework for the organization's staff to successfully implement our program model and ensure the development of new self-sustaining pediatric cardiac centers, while avoiding risk for those involved and satisfying the requirements of funders. A number of formal legal and accounting mechanisms are involved.

\section{LEGAL SUPPORT TOOL: THE PROTOCOL AGREEMENT}

Heart to Heart has no formal entity or office in Russia. This concept was explored initially but rejected because of a lack of administrative auspices.

What has taken the place of a formal entity abroad is a protocol structure. Since its inception, Heart to Heart has entered into "protocol agreements" with the authorities and interested parties with which it operates at the local and regional levels. Since the first mission, Protocol Agreements have functioned to set forth the roles and expectations of all groups involved, including outlining their responsibilities to each other and the communities they serve.

The Protocol is essentially a Memorandum of Understanding signed by the key participants in a long-term training and treatment venture. The document is the culmination of a period of research and investigation by Heart to Heart into the viability of working in a certain region.

The Protocol Agreement makes clear at the outset that "financial transactions between the parties are not involved." Then the obligations of each in the operation of the medical humanitarian venture are set forth.

In keeping with the spirit of cooperation and equal standing, the Protocol Agreement is prepared in side-by-side Russian and English versions. Because the Protocol is an agreement to cooperate in an activity, it is something less than a contract. Protocol drafting requires the focus and committed attention of authorities, professionals, and experts who will be involved directly in the project. It states commitments and requires the signatures of individuals from the entities responsible for delivering on commitments. The protocol contemplates a long-term engagement. As such, the Protocol of Agreement between the appropriate entities is probably more reliable and appropriate to a long-term humanitarian undertaking than the formation of a separate legal entity in the host country or the execution of a binding contract of specifics.

\section{NONPROFIT INDEMNIFICATION AND INSURANCE COVERAGE}

Given the particular nature of Heart to Heart's activities, including its foreign trips with medical teams and volunteers, its transportation of medical equipment and supplies to foreign locations, its employment of a full time staff, and its conducting of medical training and treatment, the organization obviously concerns itself with the adequacy of its insurance coverage and the risks of liability to its management and volunteers. Heart to Heart uses the services of a qualified insurance broker to help determine the types and levels of coverage it should carry. Coverage currently includes general liability, workers' compensation, directors and officers with employment practices, kidnap and ransom, commercial property and loss of income, and travel insurance including medical evacuation. 